\title{
Exigências Nutricionais de Caprinos da Raça Alpina em Crescimento. 1. Exigência Nutricional de Fósforo para Mantença: Perdas Endógenas e Abate Comparativo
}

\author{
Augusto César de Queiroz ${ }^{1}$, Leonardo José de Gouveia ${ }^{2}$, José Carlos Pereira ${ }^{1}$, Marcelo \\ Teixeira Rodrigues ${ }^{3}$, Kleber Tomás de Resende ${ }^{4}$, Heloisa Maria Holtz Sousa ${ }^{5}$
}

RESUMO - O objetivo deste trabalho foi determinar a exigência dietética de fósforo (P) para mantença de caprinos da raça Alpina em crescimento pelo método das perdas endógenas e pela técnica do abate comparativo. Foram usados 21 animais, machos, castrados, com idade média de quatro a seis meses e $18 \mathrm{~kg}$ PV. Destes, três foram abatidos no início do experimento, como referência, e os 18 restantes, distribuídos em tratamentos com dietas isoenergéticas e isoprotéicas, em um delineamento inteiramente casualizado com arranjo fatorial $3 \times 2$ (nível de $\mathrm{P}$ na dieta $[0,12 ; 0,21 ;$ e $0,29 \% \mathrm{MN}$ ] e forma de fornecimento da dieta [restrito ou à vontade]). O período experimental foi de 37 dias, com 30 dias para adaptação e sete dias para coleta total de fezes e urina. No final do período de coleta, três animais de cada tratamento foram abatidos. As exigências dietéticas de P para mantença foram então estimadas por meio das perdas endógenas fecal e urinária (perdas endógenas) e da retenção de P no corpo animal em relação à sua ingestão e aquele retido nos animais referência (abate comparativo). Os valores médios foram coeficiente de absorção aparente e real de $\mathrm{P},-157 \pm 30,98 \pm 10,43 \pm 24$, e $65 \pm 33 ; 235 \pm 09,145 \pm 24 \%$, respectivamente; perda diária endógena fecal e urinária de $\mathrm{P}, 8,84$ e 4,84 mg/kg PV, respectivamente; e balanço de $\mathrm{P}, 187,62 \pm 153,87$; $14,0 \pm 160,59 ; 85,96 \pm 337,85 \mathrm{mg} / \mathrm{d} ;$ e $-11,42 \pm 10,98 ; 1,04 \pm 9,14 ;-2,33 \pm 11,69 \mathrm{mg} / \mathrm{kg} \mathrm{PV}$, respectivamente. A exigência líquida diária estimada de P para a mantença foi de $13,68 \mathrm{mg} / \mathrm{kg}$ PV. As exigências dietéticas diárias de P para mantença foram estimadas em 72 e $58,46 \mathrm{mg} / \mathrm{kg} \mathrm{PV}$, pelo método das perdas endógenas e pelo abate comparativo, respectivamente.

Palavras-chave: cabras, composição corporal, exigências, fósforo, mantença, perda endógena

\section{Nutritional Requirements of Alpine Breed Goats in the Growing Phase. 1. Nutritional Phosphorus Requirement for Maintenance: Endogenous Losses and Comparative Slaughter}

\begin{abstract}
The objective of this experiment was to estimate the dietary phosphorus requirement $(\mathrm{P})$ for maintenance of kid goats in the growth phase by the endogenous loss method and comparative slaughter technique. Twenty-one males, average $18 \mathrm{~kg} \mathrm{LW}$, were used. From those, three were slaughtered at the beginning of the experiment, as reference, and the remaining 18 animals were fed isocaloric and isoproteic diets in a completely randomized design with $3 \times 2$ factorial arrangement (level of dietary P [0.12, 0.21 and $0.29 \%$ as fed] and feeding way of the diet [restricted and ad libitum]). The experimental period was 37 days, with 30-day of adaptation and seven days for total collection of feces and urine. At the end of the collection period, three animals, from each treatment, were slaughtered. The dietary requirements of $\mathrm{P}$ for maintenance were estimated the by fecal and urinary endogenous loss (endogenous loss) and the $\mathrm{P}$ retention in the animal body in relation to their intake and in that retained in the reference animal (comparative slaughter). The average values were: coefficient of apparent and real absorption of $\mathrm{P},-157 \pm 30,98 \pm 10,43 \pm 24$, and $65 \pm 33 ; 235 \pm 09,145 \pm 24 \%$, respectively; daily fecal and urinary endogenous loss of 8.84 and $4.84 \mathrm{mg} \mathrm{kg} / \mathrm{LW}$, respectively, P balance $187.62 \pm 153.87 ; 14.0 \pm 160.59 ; 85.96 \pm 337.85 \mathrm{mg} / \mathrm{d}$; and $11.42 \pm 10.98 ; 1.04 \pm 9.14 ;-2.33 \pm 11.69 \mathrm{mg} / \mathrm{kg} \mathrm{LW}$, respectively. The daily dietary P requeriments for maintenance were estimated in 72 and $58.46 \mathrm{mg} / \mathrm{kg} \mathrm{LW}$ by the endogenous loss and comparative slaughter, respectively.
\end{abstract}

Key Words: body composition, endogenous loss, goats, maintenance, phosphorus, requirements

\section{Introdução}

Os elementos inorgânicos são dieteticamente essenciais para todos os animais, exercendo influência direta sobre a eficiência de produção e correspondendo a, aproximadamente, 4 a $5 \%$ do peso corporal. Desequilíbrios minerais (deficiência ou excesso) têm sido responsáveis por problemas de baixa produção, bem como por problemas reprodutivos.

Vários métodos têm sido propostos para se estimar a exigência líquida de mantença de minerais para ruminantes, destacando-se perdas endógenas, abate

\footnotetext{
1 Professor Titular do Departamento de Zootecnia da UFV, Viçosa, MG

2 AGROCERES, Rio Claro, SP.

3 Professor Adjunto do Departamento de Zootecnia da UFV.

${ }^{4}$ Professor do Departamento de Zootecnia da UNESP, Jaboticabal, SP.

5 Mestre em Zootecnia - UFV.
} 
comparativo e uso de radioisótopos. Os resultados têm destacado o método de radioisótopos como o mais preciso, porém este método necessita laboratórios e equipamentos adequados, além de eficiente técnica de uso; o abate comparativo tem custo elevado, enquanto o método das perdas endógenas é bem simples e tem apresentado bons resultados.

As necessidades de minerais podem ser determinadas pelo método fatorial, que divide as exigências em duas etapas. Na primeira, as necessidades do mineral são obtidas a partir dos cálculos de acúmulos e excreções do mineral e de perdas endógenas inevitáveis durante o crescimento, a gestação ou a lactação, sendo o resultado expresso como exigência líquida. Na segunda, a exigência líquida é dividida pela disponibilidade do mineral nas diversas fontes dietéticas, sendo o resultado expresso como exigência dietética (AGRICULTURAL RESEARCH COUNCIL ARC, 1980). Entretanto, COELHO DA SILVA e LEÃO (1979) comentaram que os dados de disponibilidade de minerais, nas diversas fontes dietéticas, podem acarretar imprecisões nos cálculos de suas exigências dietéticas. Miller (1981), citado pelo NATIONAL RESEARCH COUNCIL - NRC (1981), relatou que a biodisponibilidade dos minerais foi mais importante que o próprio nível destes nos alimentos.

Os objetivos deste trabalho foram determinar a exigência de fósforo para a mantença de caprinos em crescimento, pelo método de perdas endógenas e do abate comparativo de animais, e verificar as possíveis relações existentes entre o consumo, a excreção fecal e urinária, a absorção aparente, o coeficiente de absorção absoluto e balanço.

\section{Material e Métodos}

\section{Experimento 1}

O experimento foi desenvolvido nas instalações do Setor de Caprinocultura do Departamento de Zootecnia da Universidade Federal de Viçosa (UFV). Foram usados vinte e um animais da raça Alpina, machos, castrados, com quatro a seis meses de idade e $18 \mathrm{~kg}$ PV, em média. Durante o período experimental, os animais foram alojados em galpão de alvenaria e mantidos em gaiolas de metabolismos individuais, providas de telas de náilon para separação das fezes e urina. Ao piso, foram adaptadas lonas plásticas e aos coletores de urina, filtros de papel, para reduzir a contaminação da urina coletada.

Os animais foram pesados, identificados e trata- dos contra ecto e endoparasitoses. Os animais foram pesados semanalmente, no início e término do ensaio de alimentação, para verificar a constância de peso durante o período experimental.

Nas Tabelas 1 e 2, são apresentados os ingredientes e os constituintes químico-bromatológicos das dietas experimentais, respectivamente. Na Tabela 2, observa-se a ocorrência de mudança apenas no ingrediente que é responsável pelo fornecimento de

Tabela 1 - Composição percentual das dietas

Table 1 - Percentage composition of the diets

\begin{tabular}{lccr}
\hline $\begin{array}{l}\text { Ingrediente } \\
\text { Ingredient }\end{array}$ & \multicolumn{3}{c}{$\begin{array}{c}\text { Nível de fósforo na dieta (\% MN) } \\
\text { Level of phosphorus in the diet (\% as fed) }\end{array}$} \\
\cline { 2 - 4 } & 0,12 & 0,21 & 0,29 \\
\hline $\begin{array}{l}\text { Capim-coastcross(feno) } \\
\text { Coastcross grass (hay) }\end{array}$ & 42,00 & 42,00 & 42,00 \\
$\begin{array}{l}\text { Sabugo de milho } \\
\text { Corn cob }\end{array}$ & 33,50 & 33,50 & 33,50 \\
$\begin{array}{l}\text { Uréia(45\%) } \\
\begin{array}{l}\text { Urea } \\
\text { Amido }\end{array}\end{array}$ & 0,82 & 0,82 & 0,82 \\
$\begin{array}{l}\text { Starch } \\
\text { Fosfato bicálcico } \\
\text { Dicalcium phosphate }\end{array}$ & 22,63 & 22,44 & 22,24 \\
$\begin{array}{l}\text { Calcário } \\
\text { Limestone }\end{array}$ & - & 0,48 & 0,95 \\
$\begin{array}{l}\text { Sal } \\
\text { Salt }\end{array}$ & 0,56 & 0,27 & - \\
\hline & 0,49 & 0,49 & 0,49 \\
\hline
\end{tabular}

Tabela 2 - Teores médios de MS, PB, EE, FDN, FDA, cinzas, $\mathrm{P}, \mathrm{Ca}, \mathrm{Mg}, \mathrm{Na}$ e $\mathrm{K}$ das dietas

Table 2 - Average contents of DM, CP, NDF, ADF, ash, Ca, Mg, $\mathrm{Na}$ e $\mathrm{K}$ of the diets

\begin{tabular}{lrrr}
\hline Item & \multicolumn{3}{c}{$\begin{array}{c}\text { Nível de fósforo na dieta }(\% \mathrm{MN}) \\
\text { Level of }\end{array}$} \\
\cline { 2 - 4 } & 0,12 & 0,21 & 0,29 \\
\hline $\mathrm{MS}(D M)(\%)$ & 86,20 & 86,65 & 86,68 \\
$\mathrm{~PB}(C P)^{1}$ & 5,53 & 5,63 & 5,33 \\
$\mathrm{EE}^{1}$ & 0,40 & 0,36 & 0,37 \\
$\mathrm{FDN}^{1}(N D F)$ & 61,25 & 60,85 & 63,60 \\
$\mathrm{FDA}^{1}(A D F)$ & 26,63 & 27,91 & 27,40 \\
$\mathrm{Cinzas}^{1}($ Ash $)$ & 3,44 & 3,48 & 3,61 \\
$\mathrm{P}^{1}$ & 0,12 & 0,21 & 0,28 \\
$\mathrm{Ca}^{1}$ & 0,58 & 0,51 & 0,52 \\
$\mathrm{Mg}^{1}$ & 0,87 & 0,88 & 0,94 \\
$\mathrm{Na}^{1}$ & 1,48 & 1,57 & 1,56 \\
$\mathrm{~K}^{1}$ & 0,67 & 0,67 & 0,70 \\
$\mathrm{Relação} \mathrm{Ca:P}_{\text {Ca:P ratio }}^{4,83}$ & 2,43 & 1,86 \\
\hline
\end{tabular}

$1 \% \mathrm{MN}$ (\% as fed).

Matéria seca (MS), proteína bruta $(\mathrm{PB})$, extrato etéreo (EE), fibra em detergente neutro (FDN), fibra em detergente ácido (FDA), cinzas.

Dry matter $(D M)$, crude protein $(C P)$, ether extract $(E E)$, neutral detergent fiber (NDF), acid detergent fiber (ADF), ash. 
fósforo, portanto, as dietas possuem os mesmos níveis de fibra e são isocalóricas, sendo adequados apenas para a mantença desta categoria animal. Inicialmente, formulou-se uma dieta para atender às exigências de mantença, segundo o NRC (1981). À esta dieta foi adicionado fosfato bicálcico, para elevar os níveis de fósforo uma (dieta 2) ou duas vezes (dieta 3) o nível recomendado.

As dietas experimentais foram fornecidas todos os dias às $7 \mathrm{~h}$ para os animais do consumo, em forma restrita, em quantidade suficiente apenas para a mantença, e às 7 e $17 \mathrm{~h}$ para os animais que receberam ração à vontade. Após ter sido estimado o consumo diário para cada animal, durante o período de adaptação, foi permitida sobra de $10 \%$ do alimento fornecido de um dia para outro, sendo, dessa forma, pesada e anotada todos os dias. Em razão dos baixos valores, as sobras foram incorporadas às rações fornecidas diariamente, permitindo ao final do período de coletas o consumo total da dieta. Este procedimento não influiu no consumo dos animais, eliminando das análises laboratoriais e estatísticas mais uma fonte que poderia contribuir com os erros experimentais.

O consumo total de matéria seca foi determinado pelo controle diário do alimento fornecido e do material rejeitado.

O período experimental teve duração de 37 dias, com 30 dias, para a adaptação dos animais ao ambiente, às bolsas de coletas de fezes e às dietas experimentais, e 7 dias de coleta de dados. Diariamente foram feitas coletas totais de fezes e urina e anotada a produção fecal e urinária. Das fezes totais coletadas, foram feitas amostragens de, aproximadamente, $200 \mathrm{~g}$ e embaladas em sacos plásticos devidamente etiquetadas e congeladas. Foram retiradas amostras de $100 \mathrm{~mL}$ da urina total coletada e, após, armazenadas em recipiente de vidro e congeladas para posteriores análises laboratoriais. Foram feitas, ainda, durante este período, amostragens de cada ingrediente da dieta e da dieta total.

As análises químicas foram efetuadas no Laboratório de Nutrição Animal do Departamento de Zootecnia da UFV, segundo metodologias descritas por SILVA (1990). As determinações das perdas endógenas foram efetuadas por meio de análises de regressão. A metodologia baseia-se nas relações entre o mineral ingerido e o mineral excretado, na forma de equação de regressão linear simples. Os valores das perdas endógenas foram obtidos extrapolando-se para o nível zero de ingestão do mineral em questão, ou seja, em que $X=0$ na equação
$\mathrm{Y}=\mathrm{a}+\mathrm{bX}$.

A estimativa da exigência líquida do mineral para mantença foi feita somando-se os valores encontrados para as perdas endógenas fecais e urinárias. Este valor foi dividido pelo coeficiente de absorção real, obtendo-se a exigência dietética para mantença, segundo o esquema fatorial proposto pelo ARC (1980).

As absorções aparentes para $\mathrm{P}, \mathrm{Ca}, \mathrm{Mg}, \mathrm{Na}$ e K, assim como seus respectivos coeficientes de absorção aparente e balanço, foram determinadas. O coeficiente de digestibilidade da matéria seca também foi determinado.

Análises de variância foram realizadas entre ingestão, excreção fecal e urinária, absorção aparente, coeficiente de absorção aparente e balanço de cada um dos macroelementos minerais, em relação aos tratamentos. Foi feita a regressão dos parâmetros excreção fecal e urinária, absorção aparente, coeficiente de absorção aparente e balanço de cada macroelemento mineral, em relação a suas ingestões. Também foram realizadas análises de regressão dos consumos, das excreções fecais e urinárias, dos coeficientes de absorção e do balanço, em relação às diferentes ingestões de P.

As análises estatísticas foram realizadas por intermédio do programa SAEG - Sistema de Análises Estatísticas e Genéticas, versão 7.X (UNIVERSIDADE FEDERAL DE VIÇOSA, 1997), de acordo com o seguinte modelo inteiramente casualizado, em arranjo fatorial $(2 \times 3)$ :

em que

$$
Y_{i j k}=\mu+D_{i}+C_{j}+D C_{i j}+e_{i j k}
$$

$Y_{\mathrm{ij}}=$ valor observado na parcela que recebeu a repetição k, do nível de $\mathrm{P}$ i no fornecimento da alimentação j;

$\mu \quad=$ efeito da média geral;

$\mathrm{D}_{\mathrm{i}} \quad=$ efeito do nível de $\mathrm{P} \mathrm{i}(\mathrm{i}=1,2,3)$;

$\mathrm{C}_{\mathrm{j}}$ = efeito do fornecimento da alimentação $\mathrm{j}$ $(\mathrm{j}=1,2,3)$;

$\mathrm{DC}_{\mathrm{ij}}=$ efeito da interação entre o i-ésimo nível de $\mathrm{P}$ e o j-ésimo fornecimento de alimentação; e

$\mathrm{e}_{\mathrm{ij}}=$ efeito dos fatores não-controlados na parcela.

\section{Experimento 2}

Vinte e um animais, machos castrados da raça Alpina, puros por origem, com quatro a seis meses idade e $20 \mathrm{~kg} \mathrm{PV}$, em média, foram usados. Destes, três animais com pesos semelhantes aos pesos dos animais distribuídos nos tratamentos foram abatidos, moídos e amostrados, servindo como animais de referência. Os 18 animais restantes foram distribuídos 
aleatoriamente nos tratamentos já mencionados no Experimento 1, sendo abatidos no final do período experimental (37 dias). Após o abate, o conteúdo do trato gastrintestinal foi retirado para determinação do peso corporal vazio (PCV); todos os tecidos foram moídos, e amostra de, aproximadamente, 500 g, retiradas. Os animais de referência foram usados para a estimativa do PCV e da composição corporal inicial do fósforo de todos os animais, segundo o método de abate comparativo descrito por LOOFGREEN e GARRETT (1968).

Posteriormente, as amostras foram armazenadas em sacos plásticos e submetidas à secagem e ao pré-desengorduramento, conforme metodologia descrita por KOCK e PRESTON (1979). Em seguida, foram moídas em moinho de bola e acondicionadas em vasilhame de vidro, hermeticamente fechado. A composição bromatológica do corpo vazio de todos os animais foi determinada.

As determinações de MS foram feitas diretamente em estufa a $105^{\circ} \mathrm{C}$, sendo, logo em seguida, extraída parte da gordura da amostra e determinada a amostra pré-desengordurada, a partir da qual se procedeu à análise bromatológica do corpo vazio. A solução mineral foi preparada e o P analisado, segundo metodologia descrita por SILVA (1990).

Foram feitas pesagens dos animais, semanalmente, no início e término do período experimental.

$\mathrm{O}$ delineamento inteiramente casualizado, em arranjo fatorial $3 \times 2$ (nível de fósforo na dieta $[0,12$; 0,$21 ;$ e $0,29 \% \mathrm{P}$ na dieta] e forma de fornecimento da dieta [restrita ou à vontade]), foi usado, com três tratamentos e seis animais por tratamento, sendo cada animal considerado uma parcela dentro dos tratamentos.

As equações de predição da composição corporal inicial de todos os animais foram obtidas por meio de equações de regressão do PV, em relação PCV, e as do logaritmo das quantidades de $\mathrm{P}$ presentes no corpo vazio, em relação do logaritmo PCV, segundo o ARC (1980). A quantidade de P mobilizada ou retida no corpo do animal, ou seja, a mobilização ou a deposição do mineral foi obtida por meio da comparação entre a composição corporal em $\mathrm{P}$, ao final do período experimental, e a composição corporal inicial.

A exigência dietética de $\mathrm{P}$ para mantença foi obtida pela relação entre $P$ retido e $P$ ingerido, quando a retenção é igual a zero em equação linear, ou seja, em que $X=0$ em equação $Y=a+b X$.

\section{Resultados e Discussão}

Na Tabela 3, encontram-se os dados obtidos paras os 18 animais submetidos aos diferentes tratamentos. Não houve efeito da interação entre o nível de $\mathrm{P}$ nas dietas e as forma de fornecimento da dieta. Pela análise da Tabela 3, pode-se inferir que não houve efeito de tratamentos para a ingestão de $\mathrm{PB}$, EE, FDN, FDA, Ca, Mg, Na e K, entretanto, obser-

Tabela 3 - Média diária da ingestão de MS, PB, EE, FDN, FDA, P, Ca, Mg, Na e K das dietas (\%MN) Table 3 - Average intake of DM, CP, EE, NDF, ADF, P, Ca, Mg, Na and $K$ of the diets (\% as fed)

\begin{tabular}{lllllll}
\hline $\begin{array}{l}\text { Ingestão } \\
\text { Intake }\end{array}$ & \multicolumn{3}{c}{$\begin{array}{c}\text { Nível de fósforo nas dietas }(\% \mathrm{MN}) \\
\text { Level of phosphorus in the diets }(\% \text { as fed })\end{array}$} & & \multicolumn{2}{c}{$\begin{array}{c}\text { Forma de fornecimento } \\
\text { Way offeeding }\end{array}$} \\
\cline { 2 - 3 } & 0,12 & 0,21 & 0,29 & & $\begin{array}{c}\text { Restrito } \\
\text { Restricted }\end{array}$ & $\begin{array}{c}\text { A vontade } \\
\text { Ad libitum }\end{array}$ \\
\hline $\mathrm{MS}^{1}(D M)$ & $31,64 \pm 4,99$ & $28,8 \pm 3,22$ & $27,0 \pm 2,14$ & & $27,5 \pm 4,14$ & $30,8 \pm 3,11$ \\
$\mathrm{~PB}^{1}(C P)$ & $2,03 \pm 0,32^{\mathrm{a}}$ & $1,9 \pm 0,21^{\mathrm{ab}}$ & $1,7 \pm 0,13^{\mathrm{b}}$ & & $1,7 \pm 0,27$ & $1,9 \pm 0,23$ \\
$\mathrm{EE}^{1}$ & $0,1 \pm 0,02^{\mathrm{a}}$ & $0,1 \pm 0,01^{\mathrm{b}}$ & $0,1 \pm 0,01^{\mathrm{b}}$ & & $0,1 \pm 0,02$ & $0,1 \pm 0,02$ \\
$\mathrm{FDN}^{1}(N D F)$ & $22,5 \pm 3,55$ & $20,2 \pm 2,26$ & $19,8 \pm 1,57$ & & $19,6 \pm 2,91$ & $22,0 \pm 1,99$ \\
$\mathrm{FDA}^{1}(A D F)$ & $9,8 \pm 1,54$ & $9,5 \pm 0,73$ & $8,5 \pm 0,68$ & & $8,8 \pm 1,19$ & $9,7 \pm 0,90$ \\
$\mathrm{P}^{2}$ & $44,0 \pm 6,95^{\mathrm{a}}$ & $69,8 \pm 7,80^{\mathrm{b}}$ & $90,4 \pm 7,18^{\mathrm{c}}$ & & $64,5 \pm 21,53$ & $71,7 \pm 20,39$ \\
$\mathrm{Ca}^{2}$ & $212,9 \pm 33,58^{\mathrm{a}}$ & $169,6 \pm 18,94^{\mathrm{b}}$ & $162,0 \pm 12,87^{\mathrm{b}}$ & & $171,0 \pm 32,25$ & $192,1 \pm 29,52$ \\
$\mathrm{Mg}^{2}$ & $319,3 \pm 50,37$ & $292,7 \pm 32,68$ & $292,9 \pm 23,26$ & & $284,4 \pm 40,83$ & $318,9 \pm 24,67$ \\
$\mathrm{Na}^{2}$ & $543,3 \pm 85,69$ & $522,2 \pm 58,30$ & $486,1 \pm 38,60$ & & $487,5 \pm 69,18$ & $546,9 \pm 46,10$ \\
$\mathrm{~K}^{2}$ & $245,9 \pm 38,79$ & $222,9 \pm 24,88$ & $218,1 \pm 17,32$ & & $215,8 \pm 31,72$ & $242,1 \pm 21,28$ \\
\hline
\end{tabular}

${ }^{1} \mathrm{~g} / \mathrm{kg} \mathrm{PV}(L W)^{2} \mathrm{mg} / \mathrm{kg} P V(L W)$.

Matéria seca (MS), proteína bruta (PB), extrato etéreo (EE), fibra em detergente neutro (FDN), fibra em detergente ácido (FDA).

Médias, na linha, seguidas por letras diferentes, diferem $(P<0,05)$ pelo teste Tukey.

Dry matter (DM), crude protein (CP), ether extract (EE), neutral detergent fiber (NDF), acid detergent fiber (ADF), ash. Means, within a row, followed by different letters, differ $(P<.05)$ by Tukey test. 
Rev. bras. zootec.

vou-se tendência de redução da ingestão de MS, à medida que se elevou o nível de P das dietas. Entretanto, BUENO (1997), CHALLA et al. (1989) e SALVIANO (1996) observaram consumos constantes de MS em dietas com diferentes níveis de P. A ingestão de PB, EE, FDN, FDA, Ca, Mg, Na e K foi semelhante às obtidas para MS, entretanto, a ingestão de $\mathrm{P}$ aumentou $(\mathrm{P}<0,05)$ com os níveis crescentes de $\mathrm{P}$ nas dietas.

O NRC (1981) preconiza valor de 2,8\% PV para ingestão de MS, no nível de mantença, portanto, semelhante ao deste estudo.

Observa-se, na Tabela 4, que as quantidades de $\mathrm{P}$ ingerido e $\mathrm{P}$ excretado aumentaram $(\mathrm{P}<0,05)$ de forma linear com a suplementação crescente do mineral. Os animais do tratamento sem suplementação externa de fósforo ingeriram menor quantidade de $\mathrm{P}$; a quantidade de $\mathrm{P}$ excretada nas fezes também aumentou em conseqüência do baixo teor de $\mathrm{P}$ presente na dieta. O NRC (1981) preconiza o valor de $0,7 \mathrm{~g} /$ dia para animais de 10 a $20 \mathrm{~kg}$ de peso vivo, para caprinos em mantença e em condições de estabulação total e mínima atividade física. Dessa maneira, as quantidades de $\mathrm{P}$ ingerido diariamente podem ser consideradas adequadas, a 60 e $130 \%$ acima das exigências para os respectivos tratamentos.

Pela análise dos resultados da Tabela 4, pode-se inferir que o suprimento de $\mathrm{P}$ foi insuficiente para $\mathrm{o}$ nível $1(0,12 \%)$, devendo o nível $2(0,21 \%)$ ser considerado suficiente, pois foi o nível em que se observou maior absorção do mineral, e o nível 3 (0,29\%), mais que suficiente para o metabolismo animal, pois todo $\mathrm{P}$

Tabela 4 - Médias da ingestão e excreção de P nas fezes e urina, absorção aparente e real balanço e relação $\mathrm{Ca}: \mathrm{P}$

Table 4 - Means of intake and $P$ excretion in the feces and urine, apparent and true absorption balance and Ca:P ratio

\begin{tabular}{|c|c|c|c|}
\hline \multirow[t]{2}{*}{ Item } & \multicolumn{3}{|c|}{$\begin{array}{l}\text { Nível de fósforo nas dietas }(\% \mathrm{MN}) \\
\text { Level of phosphorus in the diets (\% as fed) }\end{array}$} \\
\hline & 0,12 & 0,21 & 0,29 \\
\hline Ingestão de $\mathrm{P}^{1}$ & $0,67 \pm 0,13^{\mathrm{c}}$ & $1,12 \pm 0,08^{b}$ & $1,60 \pm 0,22^{\mathrm{a}}$ \\
\hline $\begin{array}{l}\text { P Intake } \\
\text { Ingestão de } \mathrm{P}^{2}\end{array}$ & $40,96 \pm 8,89^{c}$ & $65,09 \pm 8,23^{b}$ & $87,48 \pm 9,17^{\mathrm{a}}$ \\
\hline $\begin{array}{l}\text { P excretado na fezes }{ }^{1} \\
P \text { excreted in feces }\end{array}$ & $0,76 \pm 0,20^{b}$ & $1,00 \pm 0,04^{\mathrm{b}}$ & $1,58 \pm 0,38^{\mathrm{a}}$ \\
\hline $\begin{array}{l}\mathrm{P} \text { excretado na fezes }{ }^{2} \\
P \text { excreted in feces }\end{array}$ & $48,68+20,51$ & $58,23 \pm 3,46$ & $84,31 \pm 24,73$ \\
\hline $\begin{array}{l}\text { P excretado na fezes }(\%) \\
\text { Pexcreted in feces }\end{array}$ & $0,12 \pm 0,03$ & $0,12 \pm 0,01$ & $0,25 \pm 0,07$ \\
\hline P absorvido aparente ${ }^{3}$ & $-90,52 \pm 202,28$ & $115,77 \pm 121,24$ & $14,77 \pm 474,47$ \\
\hline $\begin{array}{l}P \text { apparent absorved } \\
\mathrm{P} \text { absorvido real } \\
P \text { true absorved }\end{array}$ & $61,52 \pm 237,11$ & $269,29 \pm 116,10$ & $176,83+494,77$ \\
\hline $\begin{array}{l}\mathrm{P} \text { absorvido aparente } \\
P \text { apparent absorved }\end{array}$ & $-7,72 \pm 12,50$ & $6,85 \pm 7,17$ & $3,17 \pm 19,56$ \\
\hline $\begin{array}{l}\mathrm{P} \text { absorvido real } \\
P \text { true absorved }\end{array}$ & $1,12 \pm 12,50$ & $15,69 \pm 7,17$ & $12,01 \pm 19,56$ \\
\hline $\begin{array}{l}\mathrm{P} \text { excretado na urina }{ }^{1} \\
P \text { excreted in urine }\end{array}$ & $0,10 \pm 0,09$ & $0,11 \pm 0,13$ & $0,10 \pm 0,16$ \\
\hline $\begin{array}{l}\mathrm{P} \text { excretado na urina }{ }^{2} \\
P \text { excreted in urine }\end{array}$ & $3,70 \pm 2,89$ & $5,81 \pm 7,58$ & $5,50 \pm 8,82$ \\
\hline $\begin{array}{l}\text { Balanço de } \mathrm{P}^{3} \\
\text { P balance }\end{array}$ & $187,62 \pm 153,87$ & $14,0 \pm 160,59$ & $85,96 \pm 337,85$ \\
\hline $\begin{array}{l}\text { Balanço de } \mathrm{P}^{2} \\
\text { P balance }\end{array}$ & $-11,42 \pm 10,98$ & $1,04 \pm 9,14$ & $-2,33 \pm 11,69$ \\
\hline $\begin{array}{l}\text { Relação Ca: } \mathrm{P} \\
\text { Ca:P ratio }\end{array}$ & $4,83: 1$ & $2,43: 1$ & $1,86: 1$ \\
\hline
\end{tabular}

$1 \mathrm{~g} / \mathrm{dia}(\mathrm{g} / \mathrm{d}),{ }^{2} \mathrm{mg} / \mathrm{kg} \mathrm{PV} \bullet \mathrm{d}(\mathrm{mg} / \mathrm{LW} \cdot \mathrm{d}),{ }^{3} \mathrm{mg} / \mathrm{d}$.

Médias, na linha, seguidas por letras diferentes, diferem $(P<0,05)$ pelo teste Tukey.

Means, within a row, followed by different letters, differ $(P<.05)$ by Tukey test. 
extra ingerido não foi absorvido, e sim excretado nas fezes para a manutenção da homeostase do mineral.

As quantidades diárias de $\mathrm{P}$ excretadas na urina foram de 3,$70 ; 5,81 ;$ e $5,50 \mathrm{mg} / \mathrm{kg} \mathrm{PV} /$ dia, respectivamente, para os tratamentos nos quais os animais consumiam 0,$12 ; 0,21 ;$ e $0,29 \%$ de P. Provavelmente, devido à grande variação na excreção individual, não houve diferenças entre os tratamentos. Estes valores de P na urina correspondem, em média, a 9,03; 8,93; e 6,29\% do P consumido, os quais estão bem acima dos encontrados por SALVIANO (1996) e CARVALHO (1998). A relação cálcio:fósforo dos diversos tratamentos encontra-se dentro dos valores considerados normais para ruminantes.

Para a excreção urinária de $\mathrm{P}$, em relação à ingestão, não foi encontrada diferença. Para efeito do calculo da fração urinária endógena, adotou-se a equação apresentada abaixo:

$$
\mathrm{Y}=4,84155-0,0426974 \mathrm{X} ; \mathrm{R}^{2}=0,17
$$

em que

$\mathrm{Y}=$ fósforo urinário em $\mathrm{mg} / \mathrm{kg}$ de $\mathrm{PV} / \mathrm{dia}$

$\mathrm{X}=$ ingestão de $\mathrm{P}$ em $\mathrm{mg} / \mathrm{kg}$ de $\mathrm{PV} /$ dia.

A estimativa da excreção endógena fecal foi de $8,84 \mathrm{mg} / \mathrm{kg} \mathrm{PV} /$ dia (Figura 1) e a de excreção endógena urinária, $4,84 \mathrm{mg} / \mathrm{kg} \mathrm{PV} / \mathrm{dia}$. Portanto, a exigência líquida de $\mathrm{P}$ para mantença estimada neste estudo foi de 13,68 mg/kg PV/dia.

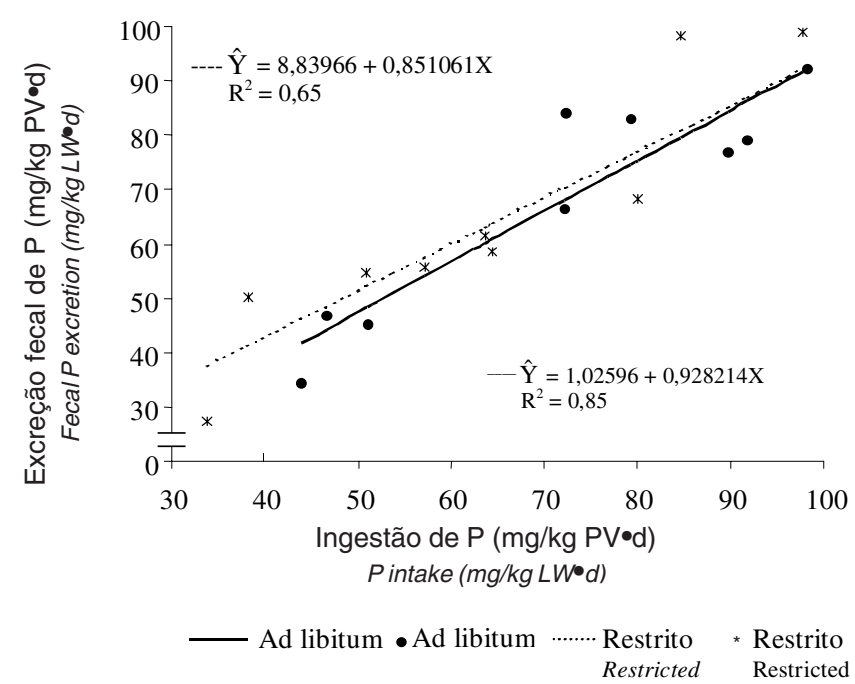

Figura 1 - Regressão da excreção fecal de $\mathrm{P}$, em relação à ingestão de $P$ pelos animais.

Figure 1 - Regression of the fecal $P$ excretion on the $P$ intake by the animals.
A Figura 1 ilustra o comportamento da curva de excreção de $\mathrm{P}$ fecal em relação à ingestão. $\mathrm{O}$ aumento na excreção de $\mathrm{P}$ nas fezes, com o incremento de $\mathrm{P}$ ingerido, foi igualmente observado por outros pesquisadores (CHALLA e BRAITHWAITE, 1988; LOUVANDINI e VITTI, 1994; BUENO, 1997; e CARVALHO, 1998).

Valores de perdas endógenas fecais de $6,87 \mathrm{mg} / \mathrm{kg}$ $\mathrm{PV} /$ dia (CARVALHO, 1998) a $56,50 \mathrm{mg} / \mathrm{kg} \mathrm{PV} \bullet \mathrm{dia}$ (SALVIANO, 1996) foram encontrados na literatura para estudos em que foram usados o $\mathrm{P}$ radioativo. Portanto, os valores das perdas endógenas relatados por meio do método fatorial ou do uso de radioisótopos são bem semelhantes, devendo-se levar em consideração que estes valores são dependentes do PV dos animais, do nível de $\mathrm{P}$ das dietas e da demanda pelo elemento.

Valores de perdas endógenas de 10,36 mg/kg PV/ dia de P para animais de 34,6 kg PV foram encontrados por BUENO (1997). CARVALHO (1998) observou valores de 11,20; 17,03; e 34,11 mg/kg PV/dia como perdas endógenas em dietas com níveis de 0,$08 ; 0,15$; e $0,38 \%$ de P na MS e em cabritos com $25 \mathrm{~kg}$ PV. Assim, a perda endógena encontrada na presente pesquisa é semelhante à verificada por outros autores, quando se usou a metodologia do $\mathrm{P}$ radioativo.

Considerando que os animais devem absorver 13,68 mg de P/kg PV•dia para suprir as perdas inevitáveis, apenas no tratamento 2, os animais absorveram quantidade suficiente de $\mathrm{P}$ para suprir estas perdas.

Os valores médios dos coeficientes de absorção aparente e real de $\mathrm{P}$, para os três níveis de $\mathrm{P}$ das dietas, encontram-se na Tabela 5.

Observou-se, na Tabela 5, que os valores encontrados para os coeficientes de absorção real de $\mathrm{P}$ (CArP) foram baixos. Valores semelhantes foram encontrados por BUENO (1997) e CARVALHO (1998), quando usaram dietas pobres em $P$ na alimentação de caprinos. Os baixos valores encontrados para os coeficientes de absorção de $P$ podem ser atribuídos à baixa qualidade da dieta. BRAITHWAITE (1985) relatou que a perda urinária de $\mathrm{P}$ em ruminante, ocasionalmente, apresenta valores altos em alguns animais, mas perda não está relacionada à ingestão de $\mathrm{P}$, e sim à eficiência de absorção maior que normal.

O baixo valor encontrado para CArP representou a baixa qualidade das dietas, ou seja, com elevado teor de fibra e baixo teor protéico. Os níveis de ingestão de $\mathrm{P}$ não influíram nos coeficientes de digestibilidade da MS, que foi de 52,58\%.

Usando-se o valor médio de CArP de 0,19 (Tabela 5), 
Rev. bras. zootec.

Tabela 5 - Médias dos coeficientes de absorção aparente (CAa) e absorção real (CAr) de fósforo para os três níveis de $P$ nas dietas

Table 5 - Means of the coefficients of apparent absorption (Caa) and true absorption (CarP) of phosphorus for the three $P$ level in the diet

\begin{tabular}{lrrr}
\hline Item & \multicolumn{3}{c}{ Nível de fósforo nas dietas (\% MN) } \\
& Level of phosphorus in the diets (\% as fed $)$ \\
\cline { 2 - 4 } & 0,12 & 0,21 & 0,29 \\
CAaP $(\%)$ & $-157 \pm 30$ & $98 \pm 10$ & $43 \pm 24$ \\
CArP $(\%)$ & $65 \pm 33$ & $235 \pm 09$ & $145 \pm 24$ \\
\hline
\end{tabular}

obteve-se a exigência dietética de $\mathrm{P}$ para mantença, $72 \mathrm{mg} / \mathrm{kg} \mathrm{PV} \bullet$ dia, quando estimada pelo método de frações endógenas, entretanto, deve-se levar em consideração a fonte de $\mathrm{P}$ usada para o cálculo da exigência dietética, pois este valor de 0,19 foi baixo e não reflete a disponibilidade de $\mathrm{P}$ das principais fontes usadas na alimentação de ruminantes.

Considerando-se que as três dietas tinham a mesma porcentagem de $\mathrm{Ca}$, porém de diferentes fontes, calcário ou fosfato bicálcico, a dieta que tinha apenas o calcário como suplementação de $\mathrm{Ca}$ foi a que apresentou melhor coeficiente de absorção aparente de $\mathrm{Ca}(\mathrm{CAaCa}$, indicando que o Ca estava mais disponível no calcário calcítico que no fosfato bicálcico (Tabela 6).

Observa-se, na Tabela 6 , efeito linear $(\mathrm{P}<0,05)$ no $\mathrm{CAaCa}$, que foi maior para os animais que tiveram maior ingestão de $\mathrm{Ca}$ (Tabela 1). Resultados semelhantes aos desta pesquisa foram encontrados por SOUSA et al. (1998), que, trabalhando com níveis crescentes de $\mathrm{Ca}$ nas dietas, encontraram coeficientes de absorção aparente crescentes com os níveis de Ca nas dietas. Contudo, os coeficientes de absorção de Ca podem estar relacionados ao fato de os animais não estarem ganhando peso e à competição pela absorção de $\mathrm{P}$, pois a absorção de $\mathrm{Ca}$ decresceu com os níveis crescentes de $P$.

Os animais que receberam os dois maiores níveis de $\mathrm{P}$ na dieta apresentaram balanço negativo do mineral, sugerindo que o nível de Ca disponível não foi suficiente para atender à exigência de mantença dos animais; o balanço de Ca foi maior $(\mathrm{P}<0,05)$ na dieta com baixo nível de P. Foi observado que houve redução na ingestão de Ca, à medida que se aumentou a ingestão de $\mathrm{P}$ (Tabela 3 ). Este comportamento ocorreu em razão da redução no consumo de MS, uma vez que os níveis de Ca na dieta foram constantes (Tabela 2). Todavia, foram observados (Figura 2) valores crescentes da excreção fecal, em função do aumento da ingestão de P; comportamento semelhante foi observado por BRAITHWAITE (1985).

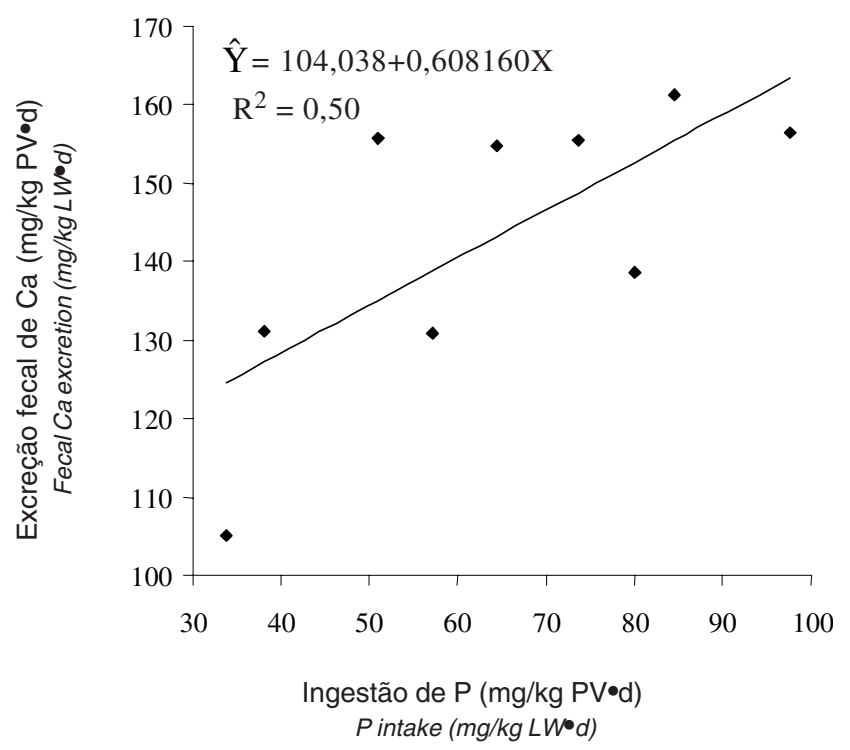

Figura 2 - Excreção fecal de $\mathrm{Ca}$, em relação à ingestão de $P$ pelos animais.

Figure 2 - Fecal escretion of Ca on the P intake by the animals.

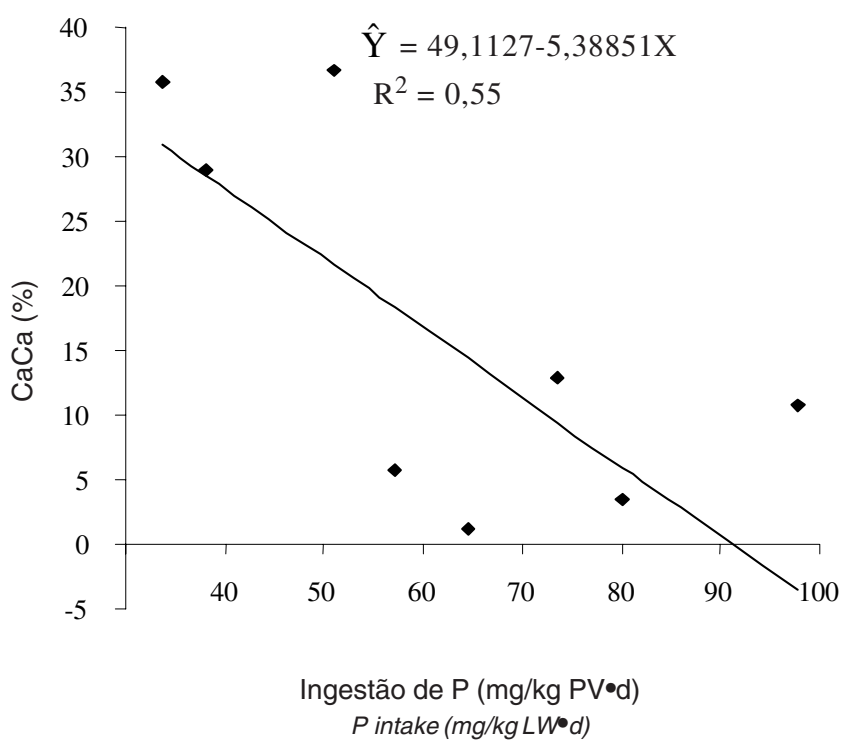

Figura 3 - Regresão do coeficiente de absorção aparente de $\mathrm{Ca}(\mathrm{CAaCa})$, em relação à ingestão de $\mathrm{P}$ pelos animais.

Figure 3 - Regression of the coefficient of apparent absorption of $\mathrm{Ca}(\mathrm{CAaCa})$ on the $P$ intake by the animals.

Pela análise da na Figura 3, constata-se que houve redução do $\mathrm{CAaCa}$ em função dos níveis crescentes de P. BRAITHWAITE (1985), trabalhando com níveis crescentes de $\mathrm{P}$ e ingestões constantes de $\mathrm{Ca}$, também observou menor coeficiente de absorção para a dieta baixa em $\mathrm{P}$, havendo redução do coeficiente de absorção aparente com o aumento dos níveis de P. 
Observa-se, na Figura 4, redução no balanço de $\mathrm{Ca}$, em função do aumento nas ingestão de $\mathrm{P}$; portanto, a redução da ingestão de $\mathrm{Ca}$ e o aumento das excreções fecais do mineral acarretaram balanço decrescente e negativo.

As excreções fecais do magnésio foram maiores $(\mathrm{P}<0,05)$ para os níveis de $\mathrm{P}$ mais elevados nas dietas, entretanto, a dieta com maior nível de $\mathrm{P}$ apresentou menor valor de excreção urinária do $\mathrm{Mg}$. Apesar de a ingestão de $\mathrm{Mg}$ ter sido igual para todas as dietas, a ingestão de $\mathrm{P}$ influenciou de forma quadrática $(\mathrm{P}<0,05)$ as excreções fecais, ocorrendo a maior $(\mathrm{P}<0,05)$ excreção do $\mathrm{Mg}$ para as dietas com os níveis maior e intermediário de $\mathrm{P}$ (Figura 5). A relação entre o coeficiente de absorção de magnésio e a ingestão de fósforo apresentou efeito quadrático $(\mathrm{P}<0,05)$, conforme ilustrado na Figura 6, o que confirma a variação ocorrida nas excreções fecais, conforme ilustrado na Figura 5.

Observa-se, na Tabela 6, que a absorção aparente do $\mathrm{Mg}$ e o seu coeficiente de absorção aparente $(\mathrm{CAaMg})$ foram maiores $(\mathrm{P}<0,05)$ para a dieta com menor nível de $\mathrm{P}$. Constatou-se que, nesta dieta, ocorreu maior $(\mathrm{P}<0,05)$ balanço deste mineral, apesar de não diferir da dieta com maior nível de P.

A absorção aparente, o coeficiente de absorção aparente, assim como o balanço de sódio e potássio, encontram-se na Tabela 6. Não houve efeito da dietas

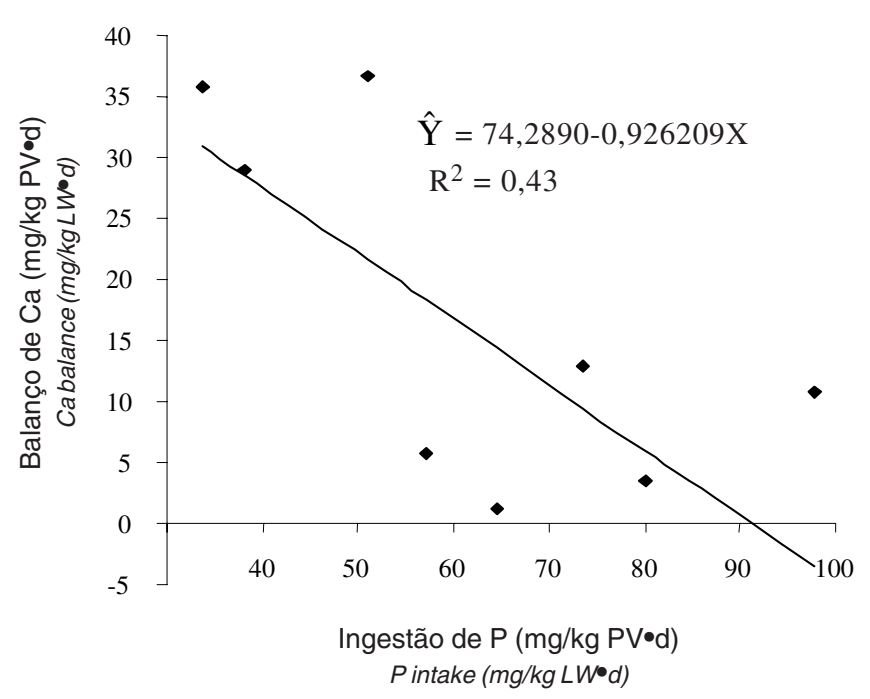

Figura 4 - Balanço de Ca em relação à ingestão de $\mathrm{P}$ pelos animais.

Figure 4 - Ca balance on the $P$ intake by the animals.

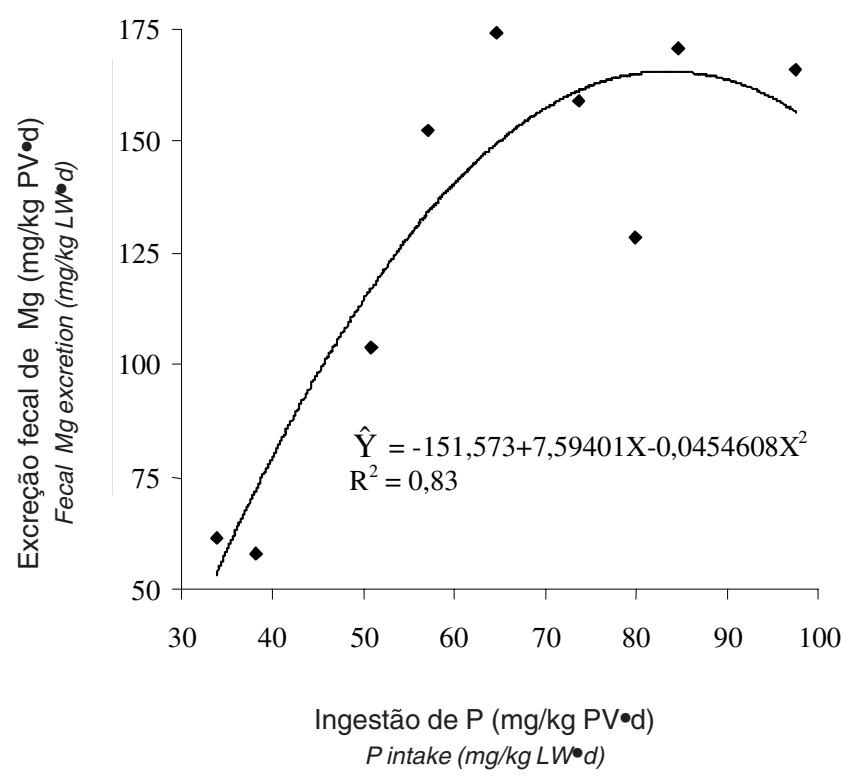

Figura 5 - Regressão da excreção fecal de $\mathrm{Mg}$, em relação à ingestão de $\mathrm{P}$ pelos animais.

Figure 5 - Regression of the fecal Mg excretion on the P intake by the animals.

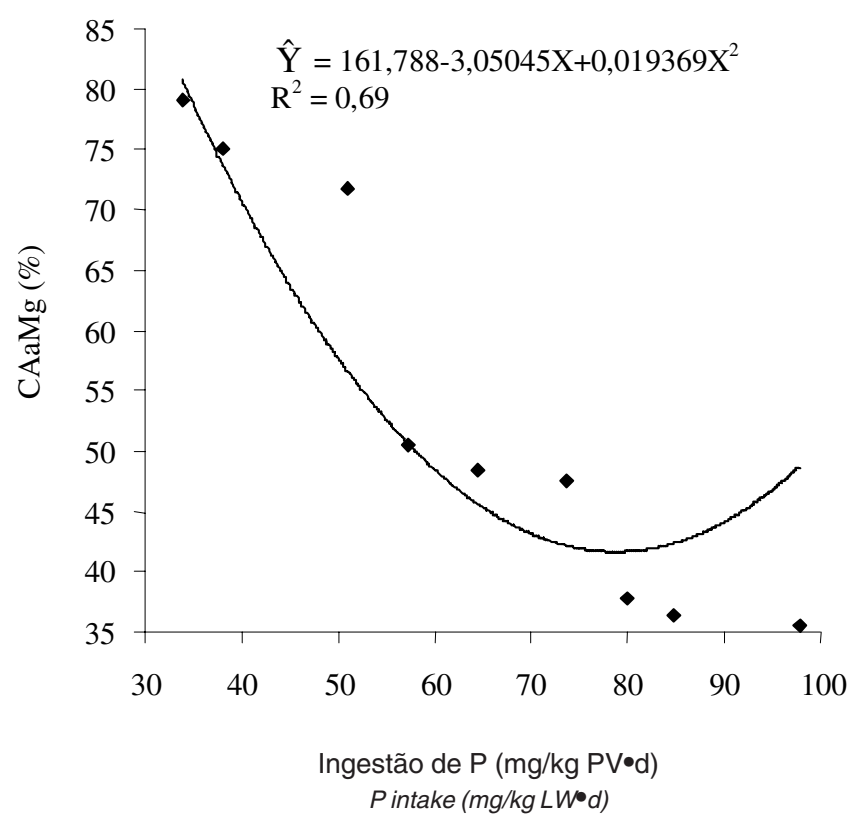

Figura 6 - Regressão do coeficiente de absorção aparente de $\mathrm{Mg}(\mathrm{CAaMg})$ em relação à ingestão de $\mathrm{P}$ pelos animais.

Figure 6 - Regression of the coefficient of apparent absorption of $\mathrm{Mg}$ (CaaMg) on the $P$ intake by the animals. 
Rev. bras. zootec.

Tabela 6 - Médias da absorção aparente (Aa), coeficientes de absorção aparente (CAa) e balanço de $\mathrm{Ca}, \mathrm{Mg}, \mathrm{Na}$ e $\mathrm{K}$

Table 6 - Means of apparent absorption (Aa), coefficients of apparent absorption (CAa) and Ca, $\mathrm{Mg}, \mathrm{Na}$ and $\mathrm{K}$ balance

Item Nível de fósforo nas dietas (\% MN)

Level of phosphorus in the diets (\% as fed)

\begin{tabular}{|c|c|c|c|}
\hline & 0,12 & 0,21 & 0,29 \\
\hline $\mathrm{AaCa}^{1}$ & $67,36 \pm 20,11^{\mathrm{a}}$ & $10,99 \pm 10,92^{b}$ & $4,75 \pm 14,09^{b}$ \\
\hline $\mathrm{CAaCa}(\%)$ & $33 \pm 10^{\mathrm{a}}$ & $7 \pm 6^{b}$ & $3 \pm 8^{b}$ \\
\hline Balanço de $\mathrm{Ca}^{1}$ & $51,53^{\mathrm{a}} \pm 15,30$ & $-\overline{2}, 16 \pm 13,87^{\mathrm{b}}$ & $-\overline{5}, 76 \pm 11,86^{b}$ \\
\hline \multicolumn{4}{|l|}{ Cabalance } \\
\hline $\mathrm{AaMg}^{1}$ & $222,53^{\mathrm{a}} \pm 40,73$ & $111,05^{\mathrm{b}} \pm 33,67$ & $128,51^{b} \pm 23,68$ \\
\hline CAaMg (\%) & $0,75^{\mathrm{a}} \pm 0,04$ & $0,40^{\mathrm{b}} \pm 0,07$ & $0,45^{\mathrm{b}} \pm 0,07$ \\
\hline Balanço de $\mathrm{MG}^{1}$ & $207,80^{\mathrm{a}} \pm 31,29$ & $95,10^{\mathrm{b}} \pm 45,43$ & $126,49^{a b} \pm 22,81$ \\
\hline \multicolumn{4}{|l|}{ Mg balance } \\
\hline $\mathrm{AaNA}^{1}$ & $361,21 \pm 80,15$ & $304,07 \pm 75,86$ & $312,48+48,22$ \\
\hline $\mathrm{CAaNa}(\%)$ & $0,71 \pm 0,03$ & $0,62 \pm 0,08$ & $0,66 \pm 0,06$ \\
\hline Balanço de $\mathrm{Na}^{1}$ & $328,26 \pm 80,75$ & $288,51 \pm 76,94$ & $299,61 \pm 47,59$ \\
\hline \multicolumn{4}{|l|}{ Na balance } \\
\hline $\mathrm{AaK}^{1}$ & $166,40 \pm 12,62$ & $112,10+46,71$ & $138,94+33,28$ \\
\hline CAaK $(\%)$ & $0,75 \pm 0,16$ & $0,53 \pm 0,16$ & $0,65 \pm 0,11$ \\
\hline Balanço de $\mathrm{K}^{1}$ & $42,80 \pm 48,83$ & $3,04 \pm 114,32$ & $65,86 \pm 29,49$ \\
\hline K balance & & & \\
\hline
\end{tabular}

$1 \mathrm{mg} / \mathrm{kg}$ PV•dia (mg/kg LW॰day).

Médias, na linha, seguidas por letras diferentes, diferem $(P<0,05)$ pelo teste Tukey.

Means, within a row, followed by different letters, differ $(P<.05)$ by Tukey test.

para os dois minerais, portanto, as pequenas variações ocorridas foram atribuídas às ingestões do mineral, em função de diferentes ingestões de matéria seca.

Embora não tenham ocorrido diferenças significativas nos valores de absorção aparente e balanço de sódio entre tratamentos, houve relação positiva entre estes parâmetros e a ingestão de sódio, mostrando que, em ingestões mais altas deste mineral, ocorre também sua maior retenção. Houve relação inversa $(\mathrm{P}<0,05)$ entre a excreção urinária de sódio e a ingestão de $\mathrm{P}$ (Figura 7), o que é atribuído à redução na ingestão de matéria seca.

Pelo método de abate comparativo, estimou-se o conteúdo corporal inicial em $\mathrm{P}$ dos animais submetidos aos tratamentos, por meio das equações de regressão obtidas com os três animais referência, a saber:

$$
\mathrm{Y}=0,030273+0,788089 \mathrm{X}\left(\mathrm{R}^{2}=99,80 \%\right)
$$

em que

$\mathrm{Y}=$ peso de corpo vazio $(\mathrm{kg})$

$\mathrm{X}=$ peso vivo $(\mathrm{kg})$.

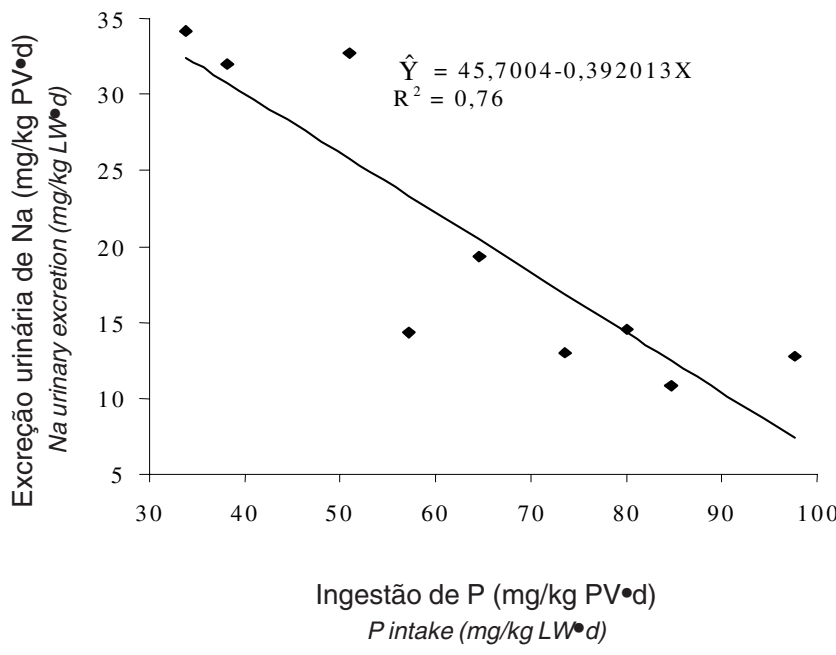

Figura 7 - Regressão da excreção urinária de $\mathrm{Na}$ em relação à ingestão de $\mathrm{P}$ pelos animais.

Figure 7 - Regression of urine Na excretion on the P intake by the animals. 
1214

$$
\mathrm{Y}=1,482509+0,582989 X\left(\mathrm{R}^{2}=56,00 \%\right)
$$

em que

$\mathrm{Y}=\mathrm{Log}$ do conteúdo em fósforo do corpo vazio $(\mathrm{g})$

$\mathrm{X}=\mathrm{Log}$ do peso de corpo vazio $(\mathrm{kg})$.

$\mathrm{Na}$ Tabela 7, encontram-se as médias da estimativa da composição corporal inicial e final e a retenção de $\mathrm{P}$ durante os 37 dias do período experimental. Observa-se que não houve efeito das dietas em relação aos conteúdos corporais inicial e final em $\mathrm{P}$, assim como na retenção corporal de P. Os resultados indicam perda corporal de $\mathrm{P}$ nas excreções endógenas, fecais e urinárias maior que a absorvida. Entretanto, essa indicação deve ser analisada com cautela, uma vez que houve redução no peso final dos animais submetidos aos tratamentos. Os animais referência, quando abatidos inicialmente, exibiram melhor condição corporal.

A análise de regressão para a quantidade de $\mathrm{P}$ retido (Y em g/37dias), em função da quantidade de $\mathrm{P}$ ingerido ( $\mathrm{X}$ em $\mathrm{mg} / \mathrm{kg} \mathrm{PV} /$ dia), pode ser expressa de acordo com a seguinte equação:

$$
\mathrm{Y}=0,178834-0,187255 \mathrm{X}\left(\mathrm{R}^{2}=9,51 \%\right)
$$

Ao se considerar $X=0$, ou seja, considerando-se zero de ingestão do mineral pelos animais, estimou-se retenção de $\mathrm{P}$ de $0,17 \mathrm{~g}$ durante o período experimental. $\mathrm{O}$ valor de $179 \mathrm{mg}$ de $\mathrm{P}$ encontrado na equação anterior, durante o período experimental, pode ser adotado com maior segurança, como retenção de $\mathrm{P}$, que os valores encontrados na Tabela 7. Isto ocorre porque: 1) a ração experimental foi calculada para a mantença, exceto o $\mathrm{P} ; 2$ ) os animais quando sofreram perda de peso para a adaptação às condições expe-

Tabela 7 - Médias do conteúdo corporal inicial e final e retenção de fósforo, durante o período experimental

Table 7 - Means of the initial and final body content and $P$ retention during the experimental period

Conteúdo corporal, g Nível de fósforos na dietas (\%MN)

Bodycontent Level of phosphorus in the diets (as fed)

\begin{tabular}{lrrr} 
& 0,12 & 0,21 & 0,29 \\
\cline { 2 - 4 } & 143,45 & 142,91 & 145,27 \\
P inicialial $P$ & 132,83 & 133,58 & 129,51 \\
P final & & & \\
Final $P$ & $-9,33$ & $-15,76$ \\
$\begin{array}{l}\text { Retenção corporal de P } \\
P \text { body retention }\end{array}$ & $-10,62$ & & \\
\hline
\end{tabular}

Médias, na linha, seguidas por letras diferentes, diferem $(P<0,05)$ pelo teste Tukey.

Means, within a row, followed by different letters, differ $(P<.05)$ by Tukey test. rimentais, principalmente à dieta experimental, não tiveram o ganho compensatório logo em seguida; e 3) como o período experimental foi relativamente curto, o peso final foi menor que o inicial.

A análise de regressão para a quantidade de $\mathrm{P}$ ingerida (Y em mg/kg PV/dia), em função do conteúdo de $\mathrm{P}$ retido ( $\mathrm{X}$ em g/37 dias), pode ser expressa, de acordo com a seguinte equação:

$$
\mathrm{Y}=58,4637-0,508042 \mathrm{X}\left(\mathrm{R}^{2}=9,51 \%\right)
$$

Assim, para retenção igual a zero $(X=0)$, ou seja, para que não haja mobilização nem deposição de $\mathrm{P}$ pelos animais, a ingestão deve ser igual a $58,46 \mathrm{mg}$ $\mathrm{P} / \mathrm{kg}$ PV. Portanto, por intermédio do método do abate comparativo, a exigência dietética de $\mathrm{P}$ para mantença foi estimada em 58,46 mg P/kg PV. Este valor foi inferior ao encontrado nesta pesquisa, pelo método das frações endógenas, cujo valor encontrado foi de $72 \mathrm{mg} / \mathrm{kg}$ PV •dia. O NRC (1981) preconizou $700 \mathrm{mg}$ P/dia para caprinos pesando entre 10 e $20 \mathrm{~kg}$, e o ARC (1980), $400 \mathrm{mg} \mathrm{P} /$ dia, nesta mesma faixa de peso. Para um animal pesando $10 \mathrm{~kg}$, estimaram-se, nesta pesquisa, as exigências de 720 e $584,60 \mathrm{mg}$ de $\mathrm{P} /$ dia, pelos métodos de frações endógenas e abate comparativo, respectivamente.

Considerando-se que, no método das frações endógenas, foi dividida a exigência de mantença pelo coeficiente de absorção real (ou disponibilidade) do P na dieta, para a determinação da exigência dietética, o maior valor encontrado por este método pode ser atribuído à baixa disponibilidade do $\mathrm{P}$.

\section{Conclusões}

Não houve diferença dos níveis de $\mathrm{P}$ sobre a ingestão e o coeficiente de digestibilidade da MS, mas houve influência dos níveis de $\mathrm{P}$ sobre a absorção, o coeficiente de absorção e o balanço aparente de $\mathrm{Ca}$ e $\mathrm{Mg}$, sendo observados valores maiores para o tratamento de menor nível de P. Para as rações contendo 0,$12 ; 0,21$; e $0,29 \%$ de $P$, foram encontrados, respectivamente, os valores médios de coeficiente de absorção de $\mathrm{P}, 0,065 ; 0,235$; e 0,145 ; excreção diária fecal de $\mathrm{P}, 48,68 ; 58,23$; e $84,31 \mathrm{mg} / \mathrm{kg}$ PV; perda urinária de $\mathrm{P}, 3,70 ; 5,81$; e $5,50 \mathrm{mg} / \mathrm{kg}$ PV; e balanço de P, $-11,42 ; 1,05 ;$ e 2,33 $\mathrm{mg} / \mathrm{kg} \mathrm{PVa}$. As perdas diárias endógenas fecal e urinária foram estimadas em 8,84 e 4,84 mg/kg PV, respectivamente. A exigência dietética diária de $\mathrm{P}$ para mantença foi estimada em 72 e $58,46 \mathrm{mg} / \mathrm{kg}$ PV pelo método das frações endógenas e do abate comparativo, respectivamente. 


\section{Referências Bibliográficas}

AGRICULTURAL RESEARCH COUNCIL - ARC. 1980. The nutrient requirement of ruminant livestock. 1.ed. London Slough: Commonwealth Agricultural Bureaux. 351p.

BRAITHWAITE, G.D. 1985. Endogenous faecal loss of phosphorus in growing lambs and the calculation of phosphorus requirements. J. Agric. Sci., 105(1):67-72.

BUENO, M.S. Níveis de fósforo para caprinos: metabolismo, Cinética e digestibilidade aparente. Piracicaba: USP, 1997. 58p. Dissertação (Doutorado em Ciências) - Universidade de São Paulo, 1997.

CARVALHO, F.F.R. Efeitos dos níveis de fósforo sobre a digestibilidade, metabolismo, perda endógena e cinética do fósforo em cabritos Saanen. UNESP: Jaboticabal, SP, 1998. 83p. Tese (Doutorado em Zootecnia) - Faculdade de Ciências Agrárias e Veterinárias/Universidade Estadual Paulista, 1998

CHALLA, J., BRAITHWAITE, G.D. 1988. Phosphorus and calcium metabolism in growing calves with special emphasis on phosphorus homoeostasis. 1. Studies of the effect of changes in the dietary phosphorus intake on phosphorus and calcium metabolism. J. Agric. Sci., 110(3):573-581.

CHALLA, J., BRAITHWAITE, G.D., DHANOA, M.S. 1989. Phosphorus homoeostasis in growing calves. J. Agric. Sci., 112(3):217-226

COELHO DA SILVA, J.F., M.I. LEÃO. 1979. Fundamentos de nutrição dos ruminantes. São Paulo: Livroceres. 380p.

KOCK, S.D.M., PRESTON, R.L. 1979. Estimation of bovine carcass composition by the urea dilution technique. J. Anim. Sci., 48(2):319-327.

LOFGREEN, G.P., GARRETT, W.N. 1968. A system for expressing net energy requirements and feed values for growing and finishing beef cattle. J. Agric.Sci., 27(3):793-806
LOUVANDINI, H., VITTI, D.M.S.S. 1994. Perda endógena de fósforo em ovinos com diferentes níveis do elemento na dieta. Pesq. Agropec. Bras., 29(1):145-149.

NATIONAL RESEARCH COUNCIL - NRC. 1981. Nutrient requirement of domestic animals: nutrient requirement of goats. Washington, D.C.: National Academic Press. 91p.

NATIONAL RESEARCH COUNCIL - NRC. 1981. Nutrient requirement of goat. Angora, dairy, and meat goat in temperate and tropical countries.1.ed. Washington, D.C.: National Academic Press. 87p.

UNIVERSIDADE FEDERAL DE VIÇOSA - UFV. 1997. SAEG - Sistema de Análises Estatísticas e Genéticas. Viçosa, MG: UFV

SALVIANO, L.M.C. Efeito de diferentes proporções de cálcio e fósforo sobre as perdas endógenas e absorção real de fósforo em ovinos. Piracicaba, SP: USP, 1996. 83p. Tese (Doutorado em Energia Nuclear na Agricultura) - Universidade de São Paulo, 1996.

SILVA, D.J. 1981. Análise de alimentos (Métodos químicos e biológicos). Viçosa, UFV. 165p.

SOUSA, H.M.H., QUEIROZ, A.C., RESENDE, K.T. et al. 1998. Exigências nutricionais de caprinos da raça alpina em crescimento. 1. Exigencia nutricional de fósforo para mantença: perdas endógenas e abate comparativo. R. Bras. Zootec., 27(1):186-192.

Recebido em: 06/04/1999

Aceito em: 24/02/2000 